\title{
The Role of Environmental Evaluation within Circular Economy: An Application of Life Cycle Assessment (LCA) Method in the Detergents Sector
}

\author{
Maria G. LUCCHETTI ${ }^{1}$, Luisa PAOLOTTI ${ }^{2 *}$, Lucia ROCCHI ${ }^{3}$, Antonio BOGGIA ${ }^{4}$ \\ ${ }^{1-4}$ Department of Agricultural, Food and Environmental Sciences, \\ Borgo XX Giugno 74, Perugia, 06121, Italy
}

\begin{abstract}
The present work aims to analyse the environmental advantages of a production process that applies circular economy. The study examines a product that is generated through the use of a certain percentage of recovered secondary materials, thus helping to avoid impacts related to the disposal of these materials and preserving the ecosystems from indiscriminate excessive natural resources extraction. The product analysed is an ecological detergent ("Ri-Detersivo" - Re-Detergent), produced by the company Tea Natura, mainly composed of regenerated vegetable oils coming from food industry. The methodology used in this paper is Life Cycle Assessment (LCA). A partial LCA will be carried out here, arriving at the saponification phase, and comparing the environmental impacts deriving from the Re-Detergent production process with those of a traditional soap, comparable to that studied in terms of function. The analysis of the case study found that the use of regenerated vegetable oils for the production of soap allows to significantly reduce the environmental impacts compared to the use of coconut oil imported from third countries.
\end{abstract}

Keywords - Circular economy; detergents; ecological products; environmental sustainability; LCA; sustainable development

\section{INTRODUCTION}

It is evident that our planet is in serious difficulty in satisfying the ever-growing demand of humanity about the use of natural resources, especially raw materials, and in absorbing the waste it produces [1], [2]. It is proved that some of the planetary limits, which define a safe operational space for human development, have already been overcome [1]. Therefore, it seems clear that the concept of sustainable development must be grounded on the balanced and rational use of resources, aiming to contrast the deterioration and depletion of the natural capital stock [3].

Most of human activities are based on a linear economic model, turning natural resources into base materials and products for sale, through a series of value-adding steps [4]. This model is regulated by the linear principle of "take-make-consume-dispose" [5], based on the incorrect assumption of an abundant availability of resources, easy to find and inexpensive to dispose of, ignoring the related environmental impacts [6]. Although some natural resources are actually renewable, the extraction dynamics and production rates dictated by human needs exceed the regenerative times of natural resources, and the environmental impacts linked to

\footnotetext{
*Corresponding author.

E-mail address: luisa.paolotti@gmail.com
} 
human activities, such as pollution and excessive waste production, exceed the capacity to absorb ecosystems [7].

Environmental issues, especially those related to the over-exploitation of natural resources, are leading the man to consider alternative solutions and new approaches, such as the circular economy, in order to achieve more sustainable and rational development models. The concept of circular economy is inspired by the observation of non-linear and complex systems, in particular of regenerative, evolutionary living systems, capable of absorbing and enhancing the contributions of negative entropy available in nature [8].

Circular economy is not fully a brand-new concept, as it based on a combination of fundamental and founding concepts such as Industrial Ecology, Regenerative Design, Natural Capitalism, Cradle to Cradle approach and Blue Economy [9]. However, the novelty is in the attention that this concept is gaining among business practitioners, consultancy firms, governments, NGO and associations, and academics [6]. Over the last 40 years, the analysis of the circular economic model has evolved considerably.

The present work aims to analyse and verify the actual environmental advantages of a production process that applies circular economy. The study examines a product that is generated through the use of a certain percentage of recovered secondary materials, thus helping to avoid impacts related to the disposal of these materials and preserving the ecosystems from indiscriminate excessive natural resources extraction.

The case study presented is about the problem of exhausted vegetable oils, as non-hazardous special waste. If improperly dispersed in the environment, these substances cause environmental, health and economic damages. The paper will be focused mainly on the environmental aspects of the problem and on the possibilities of regeneration and recycling of this waste, including its use in the soap industry.

The product analysed is an ecological detergent ("Ri-Detersivo" - Re-Detergent), produced by the company Tea Natura, mainly composed of regenerated vegetable oils coming from food industry. As we will see, the saponification of exhausted oils leads to great environmental advantages, as well as constituting a convenient choice from an economic and ethical point of view.

The methodology used in this paper is Life Cycle Assessment (LCA), that is the method of analysis of the entire life cycle of a product/process, with the aim of evaluating its main environmental impacts. The work will focus on the concepts of recycling and of "End of Waste", highlighting how these are connected with LCA methodology. LCA turns out to be an excellent tool for assessing the potential and benefits linked to the practices of recycling and the emergence of the circular economy. A partial LCA will be carried out here, arriving at the saponification phase, and comparing the environmental impacts deriving from the Re-Detergent production process with those of a traditional soap, comparable to that studied in terms of function.

\section{Exhaust Vegetable Oils}

\subsection{Exhaust Vegetable Oils: a Problematic Waste}

Transaction to a circular economy requires a cultural and structural change [7]. In particular, circular economy aims to decouple prosperity from resource consumption, i.e., consuming goods and services but not depending on extraction of virgin resources and thus ensuring closed loops, that will prevent the disposal of consumed goods in landfill [6]. Basically, an economy based on circularity is an economic model designed so that the waste 
of a given process returns to circulation, becoming a raw material for the same or for a different process [5]. Ideally, a system that has reached circularity is a system that produces no waste: integrated combinations activities act synergistically, to feed and to be fed reciprocally [6]. Systems of this type exist in nature and are ecosystems: nature does not produce waste, through cyclic processes, but it recycles and reuses matter in an infinite way, exploiting energy from the sun, wind or watercourses [7]. With continuous economic growth, the population has increased their consumption, and the environmental damage is seen to rise [10]. Therefore, the circular economy must reach the goal of imitating nature, improving and optimizing the use of the materials that enter the production systems and the energy that feeds these processes, making sure, respectively, that the value of products and materials is maintained as long as possible and using renewable energy sources.

The exhausted vegetable oils are a polluting agent, whose control and recycling are fundamental for the protection of natural ecosystems, avoiding an incorrect dispersion of this type of product within the environment. In the European Union legislation, the waste "edible oil and fat" is considered a "special non-dangerous" waste [11]. This type of waste is the result of the oxidation process of vegetable oil, which undergoes changes in its original polymeric structure, after the absorption of polluting substances coming from the carbonization of food residues, cooked or fried within it. Hence the term "exhausted", it means that the oil is no longer usable, due to the loss of its organoleptic properties [12].

The residual substance is a viscous and dense fluid, from yellow to red-brown, with an unpleasant smell, having lost all of the original purity and genuineness; it has turned into a special waste causing serious environmental problems if not properly disposed. While catering professional firms are obliged to separate waste collection of exhausted vegetable and animal oils and fats [13], which are subsequently recovered and recycled, for citizens there is no obligation to collection of used vegetable oil.

The most recent estimates indicate that in Italy, every year, 1.4 million ton nes of vegetable oil are released for consumption, divided almost equally between seed oil and olive oil, in the form of edible oil or as a component for the realization or conservation of other foods. The average per capita consumption is about $25 \mathrm{~kg}$ of vegetable oil per year. Of this amount, a residual oil percentage of about $19 \%$ is estimated, which corresponds to over 260000 tonnes of used vegetable oil, mostly in the form of frying residue. Going further to analyse this quota, it should be noted that 94000 tonnes $(36 \%)$ are produced by the catering and hospitality sector (restaurants, bars, hotels), and by commercial and industrial activities (chip shops, rotisserie laboratories, industrial catering) and the remaining 166000 tonnes (64\%) come from domestic consumption within homes [12].

Therefore, from these numbers it can be deduced that the most consistent and most critical fraction, from the management point of view, is constituted by the exhausted vegetable oil deriving from domestic activities. Moreover, the proportion of waste oils produced by the professional activities is more easily controllable and manageable, thanks to the obligations dictated by the mentioned regulations, while the real challenge is to intercept the exhausted vegetable oils produced from domestic users, who are not subject to any obligation. Often, due to the ignorance of the consequences that this act entails, citizens incorrectly dispose this type of waste. The inadequate disposal of used cooking oil entails a number of risks and problems, including serious environmental damage, as well as important economic, social and health effects. If disposed through the sewer system and discharged directly into the drains, as commonly happens in the domestic context, the exhausted oil compromises the proper functioning of the network itself (clogging the pipes) as well the functioning of purifiers, as it reduces the efficiency of biological purification. All these problems increase 
the investment and management costs of the plants, forced to adopt solutions for the separation of the oily phase from the aqueous one.

\subsection{Environmental Impact of Exhaust Vegetable Oils}

The potential environmental damages coming from inadequate disposal of exhausted vegetable oils are various, and it is also rather complex to assess the direct and indirect effects of voluntary or accidental waste oils release in the different environmental compartments.

Undoubtedly, the most evident impact concerns water pollution, which can occur either directly, if oily waste is discharged into water bodies, or indirectly, if it is introduced into the sewer system [12]. Impacts for water are connected both to the chemical nature of the oil i.e. immiscibility with water, and to the chemical compounds coming from the frying process, that are dispersed in the water bodies causing environmental and biological alterations.

The oil that reaches a body of water, due to its nonpolar character and lower density compared to water, is distributed on the surface forming a thin waterproof film, with consequent problems for aquatic environments: the oil layer on the surface inhibits gas exchange and limits the filtration of light, with strong repercussions on the ecosystems, compromising the existence of the underlying flora and fauna. It is estimated that $1 \mathrm{~kg}$ of used vegetable oil can be evenly distributed to cover an area of 1000 square meters [12].

The damage caused by oils in the soil component is also considerable. If improperly dispersed in the ground, the exhausted vegetable oil tends to form a hydrophobic film around the particles of earth and on the surface of the roots. This phenomenon creates a sort of barrier making the roots of the plants impermeable to water and, consequently, precluding at the same the intake of nutrients. Moreover, in the case in which the exhausted oil, penetrating into the ground, reaches the phreatic aquifer, it would be able to form a lentiform layer with a thickness of a few centimetres above the groundwater itself [14]. Moving with the aquifer, the oil stain could reach wells of drinking water, making this water unusable for human consumption. In fact, only one litre of oil mixed with one million litres of water is enough to alter the taste in limits that are incompatible with drinking water. For this reason, it is essential to take appropriate precautions to protect groundwater and wells, but also surface waters that may be in contact with the deeper water reserves.

The properties of exhausted vegetable oils can vary significantly in relation to their original composition and the treatments they have undergone; together with the environmental conditions, these factors influence the behaviour and consequences of release in natural environments. In many cases, exhausted vegetable oils behave similarly to mineral oils, but having been less studied as pollutants, it is sometimes more difficult to predict their consequences and act promptly. Moreover, similarly to what can happen when synthetic oils and petroleum products are dropped in aquatic environments, exhausted vegetable oils can cover the surfaces of living organisms (for example birds) with an insulating film, decreasing their ability to heat exchange and sometimes preventing their ability to move causing immobility.

The great risks associated with improper disposal of these substances lead to a careful regulation, through specific Consortiums, of oils collection, even if until now mandatory only for the professional activities. The management of waste produced by citizens is defined as "urban waste" and is therefore responsibility of the Municipalities.

\subsection{Regeneration and Recycling of Exhaust Vegetable Oils}

Many of the companies that carry out the collection of exhausted oil also deal with its storage and recovery, subjecting the waste to specific treatments that allow it to be 
transformed into a second usable raw material, to be subsequently returned to the market. The treated waste becomes an effective and convenient substitute for virgin raw materials, used in multiple processes and applications. After eliminating any food residues that may be contained, vegetable oils can be used as a renewable energy source in co-generation plants, within ecological fuels such as biodiesel. If subjected to further specific chemical or physical processes, they can be transformed into biolubricants, suitable for use in agricultural or nautical machines, as well as used as the basis of various products in the cosmetics sector, in the soap and detergent industry, in the production of fats for the tanning, of expanded clay and in the production of printing ink, as bases for paints.

The use of vegetable oils for the production of biodiesel has so far been evaluated by Italian consortium (CONOE) as the most effective and profitable; in fact, at the current moment, biodiesel and vegetable oils are at the forefront of the substitutes to petroleum-based diesel fuel in the transportation sector [15]. Biodiesel is drawing attention as a renewable and clean alternative to fossil diesel fuel because of numerous advantages such as excellent lubricity property, reduced toxicity and exhaust emissions, higher biodegradability [16]. For this reason, in the consortium system, exhausted vegetable oils sent to biodiesel production represent $90 \%$ of the total [12].

However, in this work we wanted to give space to that $10 \%$ of alternative processes that also represent excellent solutions for transforming a potentially damaging waste into a resource. In particular, the case study will deal with a process which, following treatments of regeneration and purification of exhausted vegetable oils, leads to the production of a soap.

\subsection{Use of Exhausted Vegetable Oil as Second Raw Material in the Detergents Sector}

Detergents are probably the broadest class of technical household products. The science of detergency has undergone revolutionary changes since its beginnings in the 1930s. The huge increase in detergent production has completely altered the environment and provided a large number of new materials. Depending on the composition of the detergent molecules, they have transformed our natural environment intentionally or unintentionally [17].

According to the definition reported by the Italian National Center for Chemical Substances, Cosmetic Products and Consumer Protection, "detergent is any substance or mixture containing soaps and/or other surfactants, intended for washing and cleaning activities".

That of detergents is an industrial sector with a very high influence on everyday life. In industrial countries the annual per capita consumption of detergents is of the order of 20 to $30 \mathrm{~kg}$ per year, which in Western Europe, corresponds to $4250 \mathrm{kt}$ of detergents produced. Laundry detergents represent the greatest part of this consumption. Over the years, the detergent industry has been extremely innovated, thanks to the incentives of both market trends (e.g. development of liquid or concentrated detergents) and legislation for environmental protection (reduction of phosphorus content, biodegradability, exc.).

Soap, whose discovery has ancient origins, has been the only detergent used by man for centuries. The saponification reaction consists of an alkaline hydrolysis of triglycerides (esters). These esters are the major constituents of vegetable oils and animal fats. Historically, the production of soap was a method to reuse animal fats, lard and tallow or rancid olive oil, as a way to give value to waste products coming from other processes, or to avoid throwing a precious material still potentially exploitable and recoverable, with an approach absolutely consistent with that of circular economy. 
The basic component of any modern detergent is surfactant ${ }^{\dagger}$. In general, surfactants can be divided into two categories: natural and synthetic. The difference between natural and synthetic surfactants is not in the cleaning power, but in the origin of the raw material, which can be natural (from animal fats or vegetable oils) or fossil.

In many industrial products, surfactants have actually a petrochemical origin (e.g. SLES Sodium Lauryl Sulfate), being hardly biodegradable and with an extremely high potential impact on the environment, both during extraction and refining. The need to find alternative solutions, less impacting and more sustainable than petrochemical products, has gradually led to a massive exploitation of vegetable oils, such as soy oil, coconut oil and palm oil, as natural surfactants. Table 1 shows data on the use, at global level, of the most common vegetable oils.

\begin{tabular}{lll}
\multicolumn{3}{c}{ TABLE 1. PERCENTAGE OF USE OF } \\
\multicolumn{2}{c}{ Vegetable OILS, FOR FOOD AND INDUSTRY [18] } \\
\hline Type of oil & Food use, \% & Industry use, \% \\
\hline Peanuts & 100 & 0 \\
Olive & 99 & 1 \\
Cotton & 94 & 6 \\
Sunflower & 95 & 5 \\
Soy & 80 & 20 \\
Palm tree & 72 & 28 \\
Rape & 72 & 28 \\
Coconut & 56 & 44 \\
\hline
\end{tabular}

Data in Table 1 show as the percentages of palm and coconut oil destined for industry are particularly important. Considering that these two types of oil are currently not used as sources of production of bio-fuels, it can be assumed that the high percentage that is found in the industrial sector corresponds for the most part to the soap industry.

Detergents obtained from the saponification of vegetable raw materials present real advantages from the environmental point of view, as regards the biodegradability and the preservation of fossil sources. However, there are also several negative aspects that cannot be overlooked [19]. In fact, using oils that find their best use in food may not be an ethically sustainable choice. In addition, the environmental impact and the cost that the transport of oils causes, as well as the pressure that the increasing need for these raw materials exercises on the territories of origin (deforestation) and on local populations must be considered [20].

It is easily understandable that an oil produced near where consumption takes place has less impact than one coming from third countries (countries that are not members of European Union and European Free Trade Association), sometimes very distant. It would be even better opting for waste fat substances, such as animal fats, meat-processing residues, or exhausted vegetable oils, used for storing or cooking food.

As mentioned in the previous paragraph, exhausted vegetable oils constitute a particularly problematic waste. The lack of a complete management regulation for private citizens leads in many cases to the oils throwing into dumps or illegal spreading into the environment,

\footnotetext{
$\dagger$ The surfactant molecule consists of a long non-polar chain, which is able to interact with non-polar components as fats and other impurities, and of an ionized polar part, able to interact with water molecules. This conformation explains the interaction of soap with water and fat.
} 
causing serious environmental damage [21]. If properly treated, however, these oils can be reintroduced into multiple sectors, and used as a second raw material for the production of new products, such as soaps and detergents. Therefore, the saponification of exhausted vegetable oils could be a virtuous action from an environmental point of view, but also economic and ethical. The environmental advantages mainly consist of:

- Avoiding all the problems related to disposal of a waste substance;

- Avoiding, or at least decreasing, the import of vegetable oils from abroad, reducing the environmental pressure in countries where the production of vegetable oils for industrial use is concentrated, and reducing deforestation and the use of natural resources (e.g. palm, coconut);

- Eliminating or reducing the huge environmental impact of intercontinental transport (as palm and coconut oil are mainly produced in Indonesia or Malaysia).

Avoiding the costs of managing and disposing of this waste and the costs of importing oils from third countries is also a great economic advantage. Finally, using a waste in the soap industry is a more ethically correct choice, compared to the use of vegetable oils that could constitute a valuable food resource.

Regenerating and recycling exhausted oils, reintroducing them into the production cycle of soaps, represent an excellent example of open recycling $\ddagger$. It perfectly matches one of the circular economy basic principles, according to which it is essential that everything that still has an intrinsic residual utility is not disposed of in landfills but must be valorised, recovered and reintroduced as new raw material, returning to the economic system without compromising the reserves of natural resources.

Some firms are recognizing the potential of exhausted vegetable oils in this sense, studying systems and processes in which these substances can effectively be reused. Our case study will analyse one of these processes, leading to the production of an ecological detergent ("Ri-Detersivo"), whose formula contains a good percentage of soap obtained from the saponification of post-consumption oils.

\section{Method: Life CyCle Assessment in the ConTeXt OF Circular Economy}

The approach to environmental problems through life-cycle analysis, which we can define as "Life Cycle Thinking", originated around the end of the 1960 s, when the problems related to excessive resources consumption was already beginning to be perceived [22]. The LCA methodology proposes a systemic view of production processes, through an objective assessment and quantification of energy and environmental loads and of the potential impacts associated with a product/process/activity [23], along the entire life cycle, from the acquisition of raw materials to the final disposal (so called "from cradle to grave" approach).

The term LCA was created during the SETAC congress (Society of Environmental Toxicology and Chemistry, Vermount, USA) in 1990. LCA was defined as "An objective procedure for assessing the energy and environmental loads related to a process or an activity, carried out through the identification of energy and the materials used and the waste released into the environment. The evaluation includes the entire life cycle of the process or activity, including the extraction and transportation of raw materials, manufacturing, transportation, distribution, use, re-use, recycling and final disposal" [24].

\footnotetext{
$\$$ An "Open recycling" is a recycling system in which a material arrived at the end of its life, constituting a waste, is reintegrated into a new process, different from the process that generated it.
} 
From this point of view, LCA was established as a tool allowing to quantify the environmental impacts of a product and to identify in which phase of its life cycle the most critical issues are concentrated, in order to propose the actions of improvement, leading to a decrease in the environmental impact itself [25].

The procedure was established by the international standards ISO 14040 and ISO 14044 [26], [27]; with these standards the ISO provided references for the correct application of the life cycle analysis, including the main principles and the guidelines framework for performing a LCA study. The main phases generally analysed in the LCA studies are: Goal and Scope definition, Life Cycle Inventory, Life Cycle Assessment and Interpretation [26].

The study of the life cycle of a system includes the evaluation of the environmental effects of the disposal phase. In this sense, the LCA methodology is a very effective tool for defining the best choices that aim primarily at reducing waste production and, secondly, at reducing environmental impacts connected to the "end of life" of a certain product.

A good that comes at the end of its useful life, after collection, can be used for: material recovery (re-use, recycling); energy recovery (e.g. thermal valorisation); landfill disposal. Assuming that the transfer to landfills must be considered as a last option when there are no valid recovery possibilities, each of the three alternatives has environmental aspects that must be carefully evaluated and compared with the relative benefits, in order to be able to direct the choice towards the less impactful option. In fact, it should be considered that the activities mentioned above are also industrial processes, which have their respective energy and environmental repercussions.

The principle of reuse refers to "any action by which products or components that do not constitute waste are used again for the same purpose for which they were conceived" [28]. It consists of re-using the same product, after a possible reconditioning treatment, for the same function it has carried out in its useful life cycle, without making substantial changes.

The principle of recycling, instead, concerns "every operation by which waste materials are reprocessed into products, materials, substances to be used for the original purpose or for other purposes" [28]. It consists therefore in recovering the material contained in a product and in reusing it for the production of new objects, consisting entirely or in part of the same material. Although recycling is the most developed strategy for achieving circular economy, not in all cases it leads to effective results: the energy requirements for recycling can be high, the lower quality of secondary material can lead to an increase in the demand for virgin material, or sometimes secondary materials cannot be used to replace virgin materials [29].

A limit of the recycling, which should not be underestimated, could also lie in the availability of supply chains capable of enhancing the materials. Therefore, recycling is not a goal that must be followed at all costs, rather it must be considered in combination with reduction and reuse systems, following the $3 \mathrm{R}$ principle (Reduce, Reuse, Recycle), in order to achieve sustainable management of resources.

The recycling option has to be evaluated by comparing, through an LCA approach, the energy consumption and the environmental impacts that would derive from all the activities necessary for the recovery of waste or products, with the consumption and impacts resulting from the production of the same product with virgin raw materials, and the dumping of the same waste.

Another concept, strictly connected with circular economy is that one of "end of waste" (EoW), established at European level to find a balance between the protection of the environment and the recovery of waste materials and their reintegration into production cycles. The EU Directive 2008/98/EC [28] ("Waste Framework Directive") aims to "help the 
European Union to approach a 'recycling society', trying to avoid the production of waste and to use waste as resources".

According to this Directive a waste terminates to be waste when it has undergone a recovery operation, including recycling and preparation for re-use, and meets the following conditions:

1. The substance or object is commonly used for specific purposes;

2. There is a market or an application for this substance or object;

3. The substance or object meets the technical requirements for specific purposes and complies with the existing legislation and standards applicable to that type of products;

4. The use of the substance or object will not lead to overall negative impacts on the environment or human health.

The concept of End of Waste constitutes the fulcrum on an authentically "circular" economy. In fact, the waste having the characteristics of EoW must be considered from that moment as non-waste, thus being able to be reintegrated into new production cycles.

In this perspective, the LCA methodology, highlighting the impacts caused not only by the production phase of a product, but also by the phases of use and disposal, allows to evaluate and quantify the benefits deriving from materials recycling activities, and proves to be a valid tool to support circular economy approach, promoting the transition towards a more sustainable use of resources.

\section{Case Study: LCA APPlied to the "Re-Detergent"}

\subsection{Description of the Product}

In this paragraph we report the application of the LCA method to the product "Ri-Detersivo" (Re-Detergent), an ecological detergent designed and produced by a company in central Italy (The company "Tea Natura", located in the city of Ancona - Region of Marche). This product contains a good percentage of recycled raw materials. The LCA study was carried out by using the SimaPro 8 Software [30], following the guidelines set by the ISO 14040 and ISO 14044 standards.

"Ri-Detersivo" is a detergent, both for hand washing and washing machine, that is highly concentrated, ecological and eco-sustainable, containing $35 \%$ of surfactants obtained from exhausted vegetable oils. These oils are previously recovered, filtered, deodorized, clarified and finally saponified. The "Ri-Detersivo" line was born from the will of Tea Natura to combine the concept of eco-compatibility with that of sustainability. In fact, the company uses a waste coming from post-consumption of food oil, and gives back to it the dignity of a raw material, by transforming the waste into a biodegradable surfactant.

This transformation allows eliminating the problems that these waste oils would cause in case of dispersion in the environment and, at the same time, to avoid the excessive use of fats of extra-European origin (palm, coconut, exc.) to create the surfactant.

In TEA detergents, in addition to the choice of completely avoiding surfactants of petrochemical origin such as SLES, replaced with vegetable surfactants, a total absence of other impacting agents was also reached: substances like formaldehyde, phosphorus, softeners, optical whiteners, which are ingredients with a strong and recognized ecological impact (poor biodegradability, toxicity for living aquatic organisms, presumed teratogenic action) have been avoided or replaced by more biocompatible raw materials. In particular, phosphates, enzymes or softeners have been replaced by clays, citrates and silicates; dyes and synthetic thickeners have been eliminated, and the thickening function in TEA products is 
ensured by the use of salt $(\mathrm{NaCl})$; finally, pure essential oils, where possible organic, have replaced synthetic fragrances.

Therefore, the product "Ri-Detersivo" is not only largely composed of recovery substances, that allow a considerable impacts reduction in its production phase, but also has the advantage of being more biodegradable than conventional detergents, reducing the impact in its use phase. In this way, Tea Natura succeeded in giving its personal and effective answer to two major problems: that of giving new life to a product considered waste, and that of reducing pollution due to the use of detergents.

The production of "Ri-Detersivo" therefore allows for:

- Making a highly polluting waste completely reusable, through the practice of recycling;

- Using twice a vegetable raw material of agricultural origin (first as edible oil, then as a base for soaps);

- Compensating the costs of recovery and disposal of used oils;

- Reducing the import of raw materials from third countries, resulting in a more sustainable use of resources;

- Reducing emissions of $\mathrm{CO}_{2}$ into the atmosphere, related to the transport of the above raw materials;

- Reducing the impact during the use phase, thanks to the high biodegradability of the product.

From $1 \mathrm{~kg}$ of oil is possible to obtain $17 \mathrm{~kg}$ of product. According to data collected by the Ministry of Health, every Italian annually consumes $25 \mathrm{~kg}$ of vegetable oils, and the residue is equal to about $20 \%$ of the total (about $4.5 \mathrm{~kg}$ per capita). It can be deduced that each of us would be potentially able to supply the company with the raw material necessary for the production of 76.5 bottles of laundry detergent. The production process of this detergent involves different subjects that work together in synergy to complete the product.

\subsection{Goal and Scope Definition}

\subsubsection{Goal of the Study}

The present study is a comparative LCA, i.e. it aims to study the production process of a product, in order to compare its environmental impacts with those of a product comparable in terms of function, but different in composition.

The analysis that was carried out wants to verify if the implementation of recovery and recycling operations, using a recycled raw material in a given production process, brings advantages from an environmental point of view. In particular, in this case study we would like to verify if the use of recovered exhausted vegetable oils, as a base for the production of soap, determines a reduction of the environmental impacts, compared to soaps produced only with virgin raw materials.

\subsubsection{Description of the whole Production Process of "Ri-Detersivo"}

The production process of the "Ri-Detersivo" can be divided into 5 phases:

1. Cold centrifugation;

2. Cardboard mechanical filtration;

3. Saponification: obtaining the "Soavisoap" molecule;

4. Completion of the formula by adding additional ingredients;

5. Bottling and labelling. 
In this sequence of operations, the output of a phase is totally used as input for the following phase, except for some processing scraps, which are therefore treated as waste and correctly disposed.

Phase I: Cold Centrifugation - in the first phase the basic input is the exhausted vegetable oil, which comes from a capillary collection process carried out within the territory of Marche Region by a company participating in the CONOE consortium system. The exhausted oil at the entrance looks like an emulsion of oil and water, also containing impurities due to the original function of the product, namely that of edible oil.

The oil is subjected to a cold centrifugation process, which substantially allows separating the oily phase from the aqueous and from the solid ones. At the end of this operation the main output consists of purified and clarified oil. In this phase, however, there are also some waste substances, which include water (7\% of the total), conveyed to purification plants, and organic solid ( $1 \%$ of the total) called "dust", which could be potentially reusable as a soil improver but which is currently destined to thermal destruction.

The oil resulting from this phase (with a yield of $92 \%$ ) is a product that can be recycled and reintroduced into the market as a raw material. The only other input entering this centrifugation phase is the energy required to operate the centrifuges, which, however, being a cold process, is quite small as amount.

Phase II: Cardboard mechanical filtration - the next phase is mechanical cardboard filtration. In this phase the clarified oil is subjected to a further treatment that ensures an even greater level of oil purity. The cardboard filter consists of a central body formed by plastic plates with a central hole, in which 10 cardboard filters are interposed, with a thickness of $2 \mathrm{~mm}$ each one. The oil is introduced into the filtering system through a special pump and, due to the pressure impressed by the machinery, passes from one septum to another through the cardboard filters, until it is collected in special containers. This particular type of filter allows obtaining excellent levels of purification without altering the fundamental characteristics of the filtered liquid. Also, in this case, the energy required to operate the machinery represents the only additional input. In the filtration phase, the only waste produced consists of the cardboards impregnated with oil and impurities, which must be disposed of in landfills. The oil that remains inside the cardboards causes a loss of just $2 \%$ of the incoming oil.

Phase III: Saponification - at this point of the process, the exhausted vegetable oil has been completely regenerated and is ready to be sent to the third phase of the process: the saponification. This is an important part of the process, as it is the phase in which the mixture of regenerated oils is transformed into soap. Since they are a mixture of vegetable oils, and it is almost impossible to estimate the percentage of the different oils contained in the mix (e.g. percentage of olive oil, seeds oil, exc.), a particular machinery, called dynamizer, is necessary, which allows to carry out the process in a controlled manner in order to obtain a complete and effective saponification. For this operation the Tea Natura company asked for the collaboration of a company located in the north of Italy, having such a machinery.

The inputs of this phase are regenerated waste oils from the previous phase and a percentage of coconut oil (imported from abroad), both supplied by Tea Natura. Saponification requires other ingredients such as demineralized water, caustic potash, water and acetic acid. The main output of this phase is the surfactant. As mentioned, the LCA that was carried out ends with the conclusion of this phase.

Phase IV: Completion of the formula - The fourth phase consists of completing the detergent formula, in which other types of surfactants, always of natural origin, are adde do 
the surfactant obtained from processing exhausted vegetable oils. Additionally, other substances are added to the solution such as:

- Phenoxyethanol, a preservative with low environmental impact;

- Trisodium Dicarboxymethyl Alaninate, a biodegradable sequestering agent;

- Lactic Acid, an acid of natural origin that serves as a $\mathrm{pH}$ modifier;

- Sodium Chloride, i.e. common kitchen salt, which acts as a viscosifier;

- Pure essential oils, to give a pleasant smell to the product.

Phase V: Bottling and packaging - at this point the laundry detergent is complete in all its parts and is bottled in bioplastic bottles, to which a paper label is applied. The percentage of waste oils used in the product is highlighted in the front of the package.

\subsubsection{Systems Boundaries of the LCA Study and Main Assumptions}

To focus the analysis on the production phase, an LCA 'from cradle to gate' can be performed, from the production of initial inputs to the final product, omitting the phases of product distribution [25]. It is important to point out that, at the present time, the life cycle analysis has not been applied to all the phases that lead to the production of the final Re-Detergent product, but stops at the third stage, that is the one concerning the production of soap from exhausted vegetable oils. The analysis will be completed in a second moment; the one in the current work is therefore a partial and preliminary LCA. The collection and transport of used vegetable oils, production and transport of the additional coconut oil were considered. The subsequent treatments and procedures for obtaining the soap were also taken into account. The phases of completion of the formula of the Re-Detergent, bottling and distribution, were excluded from the system. Fig. 1 shows a graphical representation of system boundaries.

Data related to capital goods and infrastructures were not taken into account in the study, since their consideration would lead to an excessive increase of the complexity of the system [27].

In reference to geographical boundaries, for data as electricity consumption the medium voltage electricity use in Italy including import from other countries was considered [31], while for natural gas the production and emission data on heat coming from gas in Europe was considered [31]. Data for transport emissions, vehicle manufacturing and maintenance represent generic European data. Data referring to the production process of the product investigated represent Italian data.

Additionally, an important consideration must be made in relation to the first phase of exhausted oils treatment: the first phase of centrifugation is a recycling process, and vegetable oils are a substance that is considered a waste, but recovered in the present process, applying in this way the concept of End of Waste above explained. For this reason, only the processes related to recovery treatments have been accounted as impacts. The upstream processes necessary for the production of these oils were therefore considered null, such as the cultivation of the plants from which the oils destined for food use were extracted, the extraction and transport of the same. These processes should instead be considered if we were using virgin raw materials. It is predictable that this factor will greatly affect, in positive terms of circular economy, the impact assessment phase and therefore the final results of the study. 


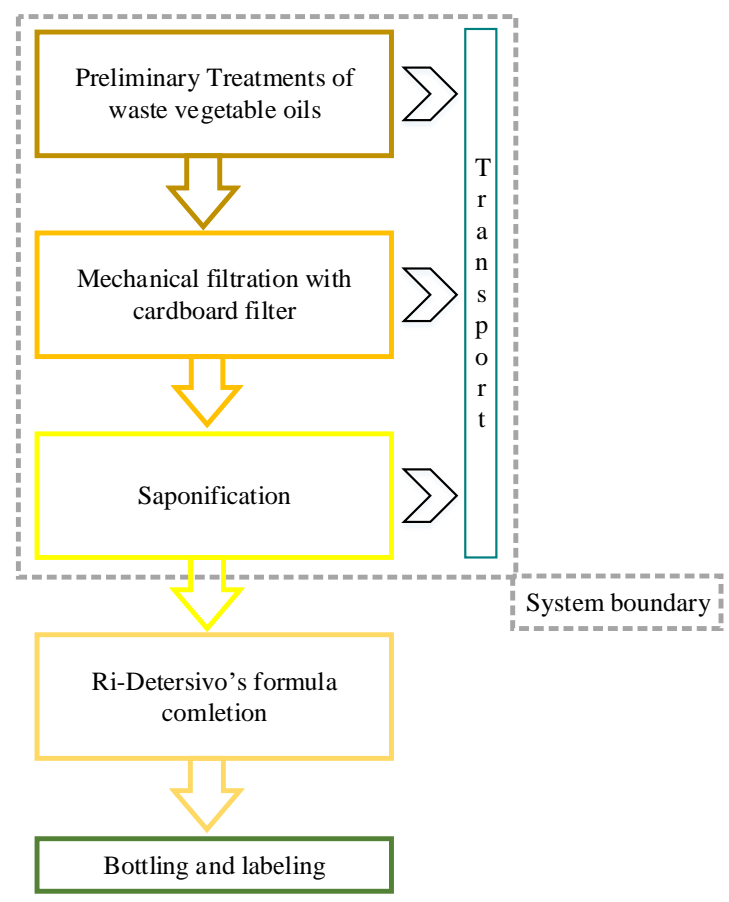

Fig. 1. Systems boundaries of the main system analysed (soap from exhausted vegetable oils).

\subsubsection{Functional Unit}

Depending on the objective and the field of application, the functional unit is defined, as a reference to which to report all the data and information collected during the LCA study [32]. All the flows in and out of the system are connected to it. The functional unit also allows for comparability of LCA results. In the specific case, it is 1 tonne of soap from recovered oils. We chose to use this quantity as functional unit, as the data about the saponification phase, supplied by the company, relate to $1000 \mathrm{~kg}$ of soap as main output.

\subsection{Inventory Analysis}

\subsubsection{Characteristics of Data}

In relation to the production process of soap obtained from used oils, the study was based, for the most part, on data supplied directly by the companies involved in the process. This type of data does not require the definition of the level of precision, since are data used by the companies as part of their ordinary management. For those data that could not be found directly, we used data available in Ecoinvent database [31]. Also, with regard to the traditional soap used as a term of comparison, the data contained in the Ecoinvent database were used, choosing an item that was as suitable as possible to the needs dictated by the purpose of the study. Other secondary data were estimated, such as those related to the transport and the flows of final waste. According to ISO, all the environmental contributions that are considered to have little relevance can be considered negligible. In this case, a "cut-off" rule of $5 \%$ was adopted [26]. 


\subsubsection{Description of the Inventory Data for each Stage}

Table 2 reports the main input and outputs of the system investigated, divided for the three phases that we considered in this context.

Collection and centrifugation of exhausted vegetable oils: the collection and centrifugation of oils is performed by a local company. This company collects and processes 2800 tonnes of exhausted vegetable oils in a year (data referring to the year 2017). As the yield is $92 \%$, it has been calculated that 2576 tonnes of oil purified by centrifugation are released from the plant annually. This product will be the main input of the next phase.

As we anticipated, among the inputs shown in Table 2 for this phase, the item "Exhausted vegetable oils" will not appear, since the contribution of this raw material in the production cycle is considered null. The evaluation of the impacts concerns only the processes related to the treatments that the oils undergo during the centrifugation.

The incoming flows are therefore the consumption of the plant in its operational phase, which substantially include consumption of gas and electricity, the first relating to the vehicles that carry out the collection and the transport of the oils, the second relative to the activity of the plant itself. Also, the transport of exhausted vegetable oils was considered, from the places where they are produced or collected, to the place of treatment, i.e. the centrifugation plant. Considering that the collecting company is mainly active in the Marche and Abruzzo regions, an average distance of $250 \mathrm{~km}$ has been estimated.

As regards the outputs of this phase, the emissions in air due to the transport of oil and to the plant operation must be considered. Also, in this case, the company provided us with the annual data for the year 2017, expressed in $\mathrm{gCO}_{2} \mathrm{eq} / \mathrm{kg}$ of treated oil. Wastewater and organic solid waste, residual of centrifugation, were counted as final waste streams. Water $(7 \%$ of the initial oil quantity) is conveyed to purification plants, while the organic solid (1\% of the initial oil quantity) is destined to thermal destruction, even if alternative solutions could be studied, such as the possibility of recycling also using it as a soil improver.

Cardboard mechanical filtration: the centrifugation phase is followed by the filtration phase using cardboard filters, which takes place within the company Tea Natura. The only material in input for this operation is represented by purified vegetable oils, the main product of the previous phase. Additionally, the energy required for the filtering system is considered. Also, in this case transports were taken into account. A distance of $50 \mathrm{~km}$, between the company that carries out the centrifugation, and Tea Natura was estimated.

The main output of this phase is purified and filtered vegetable oil. The yield is approximately $98 \%$, therefore with a starting quantity of $900 \mathrm{~kg}$ we will obtain $882 \mathrm{~kg}$ of filtered oil. In this phase there are no emissions in air, but there is the presence of a solid waste, consisting of filtering cardboards, which must be replaced after each filtering cycle, as they absorb few oils and the impurities still present in it. The machine works, each time, with a series of 10 filters, whose final weigh at the end of the process was estimated to be approximately $5 \mathrm{~kg}$.

Saponification: the saponification process is the third and last phase considered within the boundaries of the analysed system. The production of "Soavisoap" molecule takes place in a third company, located in the north of Italy (province of Bergamo). As described above, saponification is a triglyceride hydrolysis reaction promoted by alkalis: the base in this case is represented by caustic potash (potassium hydroxide), while the fat component consists mainly of regenerated vegetable oils and a lower percentage of coconut oil. These were the primary data provided by the company.

The related transports were considered. For vegetable oils, a distance of $467 \mathrm{~km}$ between Ancona and Bergamo was estimated. In relation to coconut oil, as it comes from the 
Philippines, which are one of the major producers, a travel by sea (transoceanic tanker) was assumed, with an estimated distance of $20000 \mathrm{~km}$ from the Philippines to the port of Genoa, (the main port in the North of Italy); finally, the transport of the same amount of coconut oil from the port of Genoa to Bergamo was also estimated.

TABLE 2. INPUTS AND OUTPUTS RELATED TO THE SYSTEM INVESTIGATED

\begin{tabular}{|c|c|c|}
\hline Input & Amount & Reference unit \\
\hline \multicolumn{3}{|c|}{ 1. CENTRIFUGATION PHASE ${ }^{1}$} \\
\hline Natural gas & 30000 & $\mathrm{~m}^{3}$ \\
\hline Transport, Lorry $16-32 \mathrm{t}$ & 700000 & $\mathrm{Tkm}$ \\
\hline Electricity & 54000 & $\mathrm{kWh}$ \\
\hline Output & Amount & Reference unit \\
\hline Centrifuged oil & 2576 & $\mathrm{t}$ \\
\hline $\mathrm{CO}_{2}$ emissions in air & 528752 & $\mathrm{~kg}$ \\
\hline Wastewater & 196000 & $\mathrm{~kg}$ \\
\hline Organic waste & 28000 & $\mathrm{~kg}$ \\
\hline \multicolumn{3}{|c|}{ 2. CARBOARD FILTRATION PHASE ${ }^{2}$} \\
\hline Centrifuged oil & 900 & $\mathrm{~kg}$ \\
\hline Transport, Lorry $16-32 \mathrm{t}$ & 45 & $\mathrm{Tkm}$ \\
\hline Electricity & 0.366 & $\mathrm{kWh}$ \\
\hline Purified and filtered oil & 882 & $\mathrm{~kg}$ \\
\hline Cardboard waste & 5 & $\mathrm{~kg}$ \\
\hline \multicolumn{3}{|c|}{ 3. SAPONIFICATION PHASE ${ }^{3}$} \\
\hline Treated and filtered oil & 200 & $\mathrm{~kg}$ \\
\hline Transport filtered oil & 93.4 & $\mathrm{Tkm}$ \\
\hline Crude coconut oil & 20 & $\mathrm{~kg}$ \\
\hline Transport coconut oil (sea) & 400 & $\mathrm{Tkm}$ \\
\hline Transport coconut oil (road) & 4 & $\mathrm{Tkm}$ \\
\hline Water & 770 & $\mathrm{~kg}$ \\
\hline Potassiumhydroxide $(\mathrm{KOH})$ & 99 & $\mathrm{~kg}$ \\
\hline Electricity & 200000 & $\mathrm{kWh}$ \\
\hline Output & Amount & Reference unit \\
\hline "Soavisoap" molecule & 1000 & $\mathrm{~kg}$ \\
\hline Water & 100 & $\mathrm{~kg}$ \\
\hline
\end{tabular}

$\overline{{ }^{1} \text { Data related to } 2800 \mathrm{~kg} \text { of treated oil; }{ }^{2} \text { Data related to } 900 \mathrm{~kg} \text { of treated oil; }{ }^{3} \text { Data related }}$ to $1000 \mathrm{~kg}$ of "Soavisoap". Source: our elaboration from primary company data. 
The reaction takes place in aqueous solution, therefore, among the inputs, a certain quantity of water is necessary. Another reagent in the saponification mixture is Trilon $M$ (Methylglycinediacetic acid, MGDA), a biodegradable complexing agent. However, since this substance was not present in the software database and since it is present in small quantities $(2.4 \mathrm{~kg})$ compared to the other components of the reaction, it was considered possible to neglect this input. Among the outputs, in addition to the main output $(1 \mathrm{t}$ of "Soavisoap", i.e. our functional unit), a consumption of water, due to the reactor washing water, must be considered.

\subsection{Impact Assessment Results}

Analysing the life cycle of "Soavisoap", we found that the purified oil, derived from exhausted vegetable oils, affects the entire cycle of product life, for only $2.7 \%$. This impact is not even shown in the results, because inferior to $5 \%$, which was the threshold of significance set at the beginning of the analysis for showing or not the impacts. It means that the phase of purification of the exhausted oils is really few impacting, in comparison with the rest of the processes considered in the system.

The results of the impact assessment phase (Method: EcoIndicator 99 - [33]) are shown in the graph below. In particular, Fig. 3 shows the impacts related to the entire life cycle of the "Soavisoap" product, grouped into impact categories (Normalization". phase).

Moreover, the comparison between the "Soavisoap" product and a generic coconut oil-based soap, chosen from the Ecoinvent database, has been performed. Table 3 shows the numerical values relating to the normalization phase of the comparison of 1 tonne of "Soavisoap" with 1 tonne of standard coconut oil soap.

The results show that the analysed production process causes impacts mainly in three categories: Land Use (Damage caused by occupation and reconversion of the territory), included into the macro-category "Ecosystem quality"; Fossil Fuels (damage caused by the extraction and consumption of fossil fuels), included within the macro-category "Exploitation of resources"; Respiratory effects - inorganics (Respiratory damage caused by organic substances in air), related to the macro-category "Human health". Among these impact categories, the one that has the greatest relevance is that related to the use of the land: this high impact, in fact, is caused by the process related to production and use of coconut oil, even if is used in minimum quantities, because of the problem of reconversion of territories for the cultivation of the palm from which it is extracted. The high impact obtained in relation to coconut oil underlines the importance of reducing as much as possible the use of virgin row materials in these types of products, being their use very impacting for the natural system.

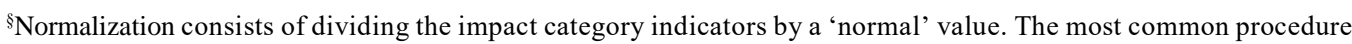
to ascertain the normal value is to determine the impact category indicators for a region during an entire year and divide this result by the number of inhabitants in that area. Therefore, the final results are expressed in points; the higher the score, the more important the impact is.
} 


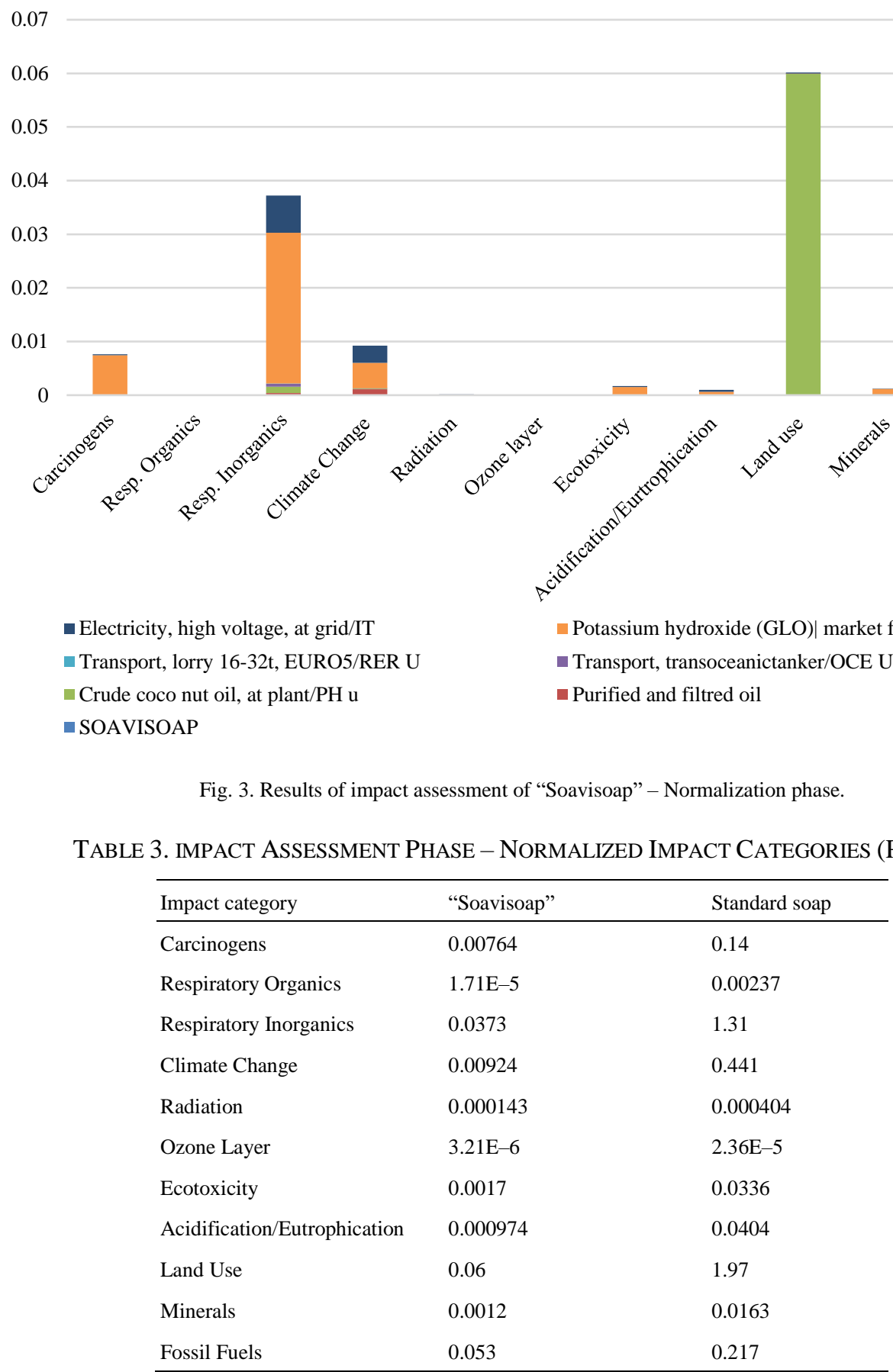


A not expected result was the high weight in the fossil fuels and respiratory inorganics categories given by potassium hydroxide production. The remaining contribution for the same categories is given by the consumption of electricity and the processes necessary for the production of soap. Using renewable energies could mitigate the impact given by electricity consumption in the production of the soap.

A greater contribution was expected for what concerns transport; however, the damage due to transport appears to be minor compared to the major causes of impact described above. All the remaining categories appear to have less significant impacts, as concerning the normalization phase results.

As regards the comparison between the production process of "Soavisoap" and the standard soap originating from coconut oil, the following can be deduced: the "Soavisoap" causes impacts extremely lower than standard soap, with reference to all impact categories. In particular, the biggest difference between the two products, in favour of the "Soavisoap", is found in the categories Land use, Respiratory Inorganics, Climate Change and Fossil Fuels (Table 3). With regard to the Land use category, this result is due to the fact that in "Soavisoap", the use of coconut oil is largely replaced by the use of regenerated vegetable oils, whose impact related to the production of oil itself must be considered null, as previously explained; this greatly reduces the impacts related to this category. This result is highlighted in Table 3, where the item "Land use" has a value of 0.06 Points for the "Soavisoap", against a value of 1.97 Points related to coconut oil soap. Also, the remaining categories (Carcinogens, Respiratory Organics, Radiation, Ecotoxicity, Ozone Layer, Acidification/Eutrophication, Minerals) result being sensibly lower in the "Soavisoap".

This confirms that, re-using vegetable oils, and avoiding using virgin row materials, allows minimizing impacts in each of the areas related to human health, ecosystem preservation and consumption of resources.

\section{Conclusion And Future Perspectives}

The purpose of this work was to demonstrate the potential of recycling practices, as a way for a transition to the new circular economy model. In relation to the comparative analysis carried out, the hypothesis that a product containing raw materials of secondary origin could have a lower environmental impact, compared to an equivalent standard product, was confirmed.

The analysis of the case study found that the use of regenerated vegetable oils for the production of soap allows to significantly reducing the environmental impacts compared to the use of coconut oil imported from third countries. This highlights the importance of focusing on the use of "zero $\mathrm{km}$ " raw materials and the search for solutions aimed at upgrading the waste that is produced in the national territory. Moreover, along with the upgrading of waste, this work would like to emphasize the need for the waste production reduction as first priority.

It is important to highlight that for making the analysis relating to the case study, assumptions and simplifications were made. However, the results obtained and presented can be considered a simplified representation of reality. Therefore, the work can be considered a good basis for affirming that, in certain contexts, recycling can lead to real environmental benefits.

This does not mean that recycling is the solution to current environmental problems. As mentioned, recycling is not always a convenient solution; for this reason, an evaluation must be carried out, that foresees the consequences and compares it with other possible 
alternatives. In any case it is not a valid solution alone. It is clear that without changing attitudes towards the consumption of resources, towards the dynamics of consumerism that prevail today, recycling itself loses its meaning. It is necessary to accompany this practice with a gradual reduction in the consumption of goods and resources, in order to lead to a real improvement.

In this context, it would be interesting to carry out other types of analysis, such as for example contingent analysis, to study other aspects of the issue, as what is the degree of knowledge of people on the subject and what is the perception of direct consumers towards the product analysed, but also towards other products that contain recycled material within them.

In this sense, Tea Natura, together with the companies with which it collaborates, is committed to organizing awareness campaigns and environmental education activities, participating in fairs and involving schools, so as to spread knowledge about the problem of waste and the concept of recycling, showing and distributing a product, the Re-Detergent, which is in fact a concrete example of the synthesis of these two concepts.

In conclusion, by conveying our choices and our purchases towards products like the one analysed, we can directly facilitate the transition to the circular economy and encourage those realities that have already begun to move in this direction. Finally, we can indirectly contribute to reducing the environmental impact on ecosystems and to limit that series of negative environmental events and trends that seem at the moment unstoppable.

\section{ACKNOWLEDGEMENT}

We would like to thank the company Tea Natura, his owner Piero Manzotti for his great support in this work and availability in providing data. Also, we would like to thank the company Adriatica Oli and Dr. Pierpaoli of "Pierpaoli Eco Bio" for their kind support in providing other primary data for the LCA study.

\section{REFERENCES}

[1] Steffen W., Broadgate W., Deutsch L., Gaffney O., Ludwig C. The trajectory of the Anthropocene: The Great Acceleration. The Anthropocene Review 2015:2:81-98. doi:10.1177/2053019614564785

[2] FAO. Statistical yearbook 2013. Rome, 2013.

[3] Henckens M. L. C. M., Ryngaert C. M. J., Driessen P. P. J., Worrell E. Normative principles and the sustainable use of geologically scarce mineral resources. Resources Policy 2018:59:351-359. doi:10.1016/j.resourpol.2018.08.007

[4] Stahel W. R. The circular economy. Nature 2016:531:435-438. doi:10.1038/531435a

[5] Ellen Macarthur Foundation. Towards the Circular Economy, Vol. 1: Economic and Business Rationale for a Circular Economy. Cowes: Ellen Macarthur Foundation, 2012.

[6] Sauvé S., Bernard S., Sloan P. Environmental sciences, sustainable development and circular economy: Alternative concepts for trans-disciplinary research. Environmental Development 2016:17:48-56. doi:10.1016/j.envdev.2015.09.002

[7] Ghisellini P., Cialani C., Ulgiati S. A review on circular economy: the expected transition to a balanced interplay of environmental and economic systems. Journal of Cleaner Production 2016:114:11-32. doi:10.1016/j.jclepro.2015.09.007

[8] Lombardini G. Visions of sustainability. Environmental policies and instruments of evaluation (Original title: Visioni della sostenibilità. Politiche ambientali e strumenti di valutazione). Milano: Franco Angeli, 2016.

[9] Saidani M., Yannou B., Leroy Y., Cluzel F. How to Assess Product Performance in the Circular Economy? Proposed Requirements for the Design of a Circularity Measurement Framework. Recycling 2017:2(1):6. doi:10.3390/recycling2010006

[10] Sutthichaimethee P., Ariyasajjakorn D. Forecast of Carbon Dioxide Emissions from Energy Consumption in Industry Sectors in Thailand. Environmental and Climate Technologies 2018:22:107-117. doi:10.2478/rtuect-2018-0007 
[11] 2000/532/EC: Commission Decision of 3 May 2000 replacing Decision 94/3/EC establishing a list of wastes pursuant to Article 1(a) of Council Directive 75/442/EEC on waste and Council Decision 94/904/EC establishing a list of hazardous waste pursuant to Article 1(4) of Council Directive 91/689/EEC on hazardous waste (notified under document number C(2000) 1147) (Text with EEA relevance).

[12] National Consortium of collection and treatment of vegetable and animal exhausted oils and fats. Annual Report, 2018.

[13] D. Lgs. April 3 2006, n. 152. Environmental Norms. Official Italian Gazette 2006:8.

[14] Udo E. J., Fayemi A. A. A. The Effect of Oil Pollution of Soil on Germination, Growth and Nutrient Uptake of Corn1. Journal of Environmental Quality 1975:4:537-540. doi:10.2134/jeq1975.00472425000400040023x

[15] Gulum M., Onay F.K., Bilgin A. Evaluation of Predictive Capabilities of Regression Models and Artificial Neural Networks for Density and Viscosity Measurements of Different Biodiesel-Diesel-Vegetable Oil Ternary Blends. Environmental and Climate Technologies 2018:22(1):179-205. doi:10.2478/rtuect-2018-0012

[16] Gulum M., Bilgin A. An Experimental Optimization Research of Methyl and Ethyl Esters Production from Safflower Oil. Environmental and Climate Technologies 2018:22(1):132-148. doi:10.2478/rtuect-2018-0009

[17] Aboul Kassim T. A., Simoneit B. R. T. A review of the nature, chemistry, and behavior in the aquatic environment. Part I. Chemical composition and analytical techniques, 2009.

[18] Foreign Agricultural Service, Official USDA Estimates, 2018.

[19] Taylor A. Soaps and detergents and the environment. Journal of the American Oil Chemists' Society 1980:57:859-861. doi:10.1007/BF02687678

[20] Vijay V., Pimm S. L., Jenkins C. N., Smith S. J. The Impacts of Oil Palm on Recent Deforestation and Biodiversity Loss. PloS ONE 2016:11(7): e0159668. doi:10.1371/journal.pone.0159668

[21] Pandey R. A., Sanyal P. B., Chattopadhyay N., Kaul S. N. Treatment and reuse of wastes of a vegetable oil refinery. Resources, Conservation and Recycling 2003:37(2):101-117. doi:10.1016/S0921-3449(02)00071-X

[22] Baldo L. G., Stefano R., Marino M. Life Cycle Analysis. The instruments for sustainable design of materials, products and processes (Original title: Analisi del ciclo di vita LCA. Gli strumenti per la progettazione sostenibile di materiali, prodotti e processi). Milano: Edizioni Ambiente, 2005.

[23] Rocchi L., Paolotti L., Fagioli F. F., Boggia A. Production of insulating panel from pruning remains: an economic and environmental analysis. Energy Procedia 2018:147:145-153. doi:10.1016/j.egypro.2018.07.044

[24] SETAC. Guidelines for Life-Cycle Assessment: a code of practice. Bruxell: SETAC, 1993.

[25] Paolotti L., Boggia A., Castellini C., Rocchi L., Rosati A. Combining livestock and tree crops to improve sustainability in agriculture: a case study using the Life Cycle Assessment (LCA) approach. Journal of Cleaner Production 2016:131:351-363. doi:10.1016/j.jclepro.2016.05.024

[26] International Organization for Standardization, 2006a. Environmental Management - Life Cycle Assessment: Principles and Framework. ISO 14040.

[27] International Organization for Standardization, 2006b. Environmental management - Life cycle assessment: Requirements and Guidelines. ISO 14044.

[28] Directive 2008/98/EC of the European Parliament and of the Council of 19 November 2008 on waste and repealing certain Directives.

[29] Haas W., Krausmann F., Wiedenhofer D., Heinz M. How Circular is the Global Economy? An Assessment of Material Flows, Waste Production, and Recycling in the European Union and the World in 2005. Journal of Industrial Ecology 2005:19:765-777.

[30] Product Ecology Consultants. SimaPro LCA Software. Plotterweg, 1990.

[31] Ecoinvent. Ecoinvent Data V2.2. Swiss Centre for Life Cycle Inventories, 2010.

[32] Rocchi L., Kadziński M., Menconi M. E., Grohmann D., Miebs G., Paolotti L., Boggia A. Sustainability evaluation of retrofitting solutions for rural buildings through life cycle approach and multi-criteria analysis. Energy and Buildings 2018:173:281-290. doi:10.1016/j.enbuild.2018.05.032

[33] Goedkoop, M., Spriensma, R. The Eco-indicator 99 e a Damage Oriented Method for Life Cycle Impact Assessment. Methodology Report, 3 ed. Plotterweg: Product Ecology Consultants, 2001. 